\title{
Nonparametric Estimation of the Interval Reliability
}

\author{
Angel Mathew* \\ Department of Statistics, Maharajas College, \\ Ernakulam, Kerala 682 011, India \\ N. Balakrishna \\ Department of Statistics, Cochin University of Science and Technology, \\ Cochin, Kerala 682 022, India \\ E-mail: nb@cusat.ac.in \\ Received 4 september 2013 \\ Accepted 12 May 2014
}

\begin{abstract}
The interval reliability of a repairable system is the probability that the system is operating at a specified time and will continue to operate for a specified interval of time. This quantity is especially important for equipment, which must be working when an emergency situation arises. The present paper discusses the nonparametric estimation of the interval reliability when (i) the data on ' $n$ ' complete cycles of system operation are available, (ii) the data are subject to right censorship, and (iii) the process is observed up to a specified time ' $T$ '. A Simulation study is conducted to assess the performance of the estimators. The proposed method is also illustrated using a compressor failure data.
\end{abstract}

Keywords: Availability; Censored data; Empirical distribution function; Product limit estimator; Renewal function.

\section{Introduction}

Consider a repairable one-unit system which is being activated and functioning at time $t=0$. Suppose that the state of the system at any time is either 'up' or 'down'. We interpret the 'up' state as the system is functioning and the 'down' state as the system is not functioning and undergoing repair after failure. The first 'up' and 'down' states together constitute the first cycle of the system. If we define

$$
\xi(t)=\left\{\begin{array}{ll}
1 & \text { if the system is functioning at time } t \\
0 & \text { otherwise }
\end{array},\right.
$$

then $\xi(t)$ represents the state of the system at time ' $t$ '. Based on $\xi(t)$ a number of useful measures of the system availability may be constructed. Some important measures of the system availability are (i) the point availability, $A(t)=P[\xi(t)=1]$; (ii) the average availability, $A_{a v g}(t)=\frac{1}{t} \int_{0}^{t} A(u) d u$ and (iii) the limiting availability, $A=\lim _{t \rightarrow \infty} A(t)$ provided the limit exists; See Barlow and Porschan (1975). The properties of these measures are usually studied using the successive failure and repair times of the system.

\footnotetext{
*Correspondence: Angel Mathew, Department of Statistics, Maharajas College, Ernakulam, Kerala 682 011, India. E-mail: angelmathew@gmail.com
} 
The estimation of various measures of the system availability has been studied extensively in recent years. The nonparametric point and interval estimation of the point availability has been discussed by Baxter and $\mathrm{Li}$ (1994) and $\mathrm{Li}$ (1999) in the case of complete and censored observations respectively. Ouhbi and Limnios (2003) constructed a nonparametric confidence interval for the point availability as a special case of Semi-Markov process. Balakrishna and Mathew (2009) studied the nonparametric point and interval estimation of the average availability. Since it is difficult to obtain closed form expressions for the point and average availability, except for few simple cases, in the literature more attention is being paid to the estimation of the limiting availability; see, for example, Mi (1995, 1999), Baxter and Li (1996), and Abraham and Balakrishna (2000).

In the context of repairable system, another important measure of successful performance of a system is the interval reliability. The interval reliability, $R(x, t)$ is defined as the probability that at a specified time ' $t$ ' the system is operating and will continue to operate for an interval of duration ' $x$ '; see, e.g., Barlow and Hunter (1961). That is, $R(x, t)=P[\xi(s)=1, t \leq s \leq t+x]$. The interval reliability becomes simply reliability when $t=0$ and point availability at time ' $t$ ' as $x \rightarrow 0$. Thus, the interval reliability is one of the most important measures of system performance from the viewpoint of reliability and availability, and is useful in many practical situations. A typical example is the model of a standby generator, in which ' $t$ ' is the time until the electric power stops and ' $x$ ' is the required time until the electric power recovers again. In this case, the interval reliability represents the probability that a standby generator will be able to operate during the interruption of the electric power (cf., Nakagawa 2005).

Let $\left\{X_{n}\right\}$ and $\left\{Y_{n}\right\}$ be independent sequences of independent and identically distributed (i.i.d.) non-negative random variables with common distribution functions $F_{X}\left(\right.$.) and $F_{Y}($.$) respectively. Assume that F_{X}\left(\right.$.) and $F_{Y}($.) have positive means $\mu_{X}$ and $\mu_{Y}$ respectively. Define $Z_{n}=X_{n}+Y_{n}$ and let $F_{Z}($.) be its distribution function.

Let $S_{n}=\sum_{i=1}^{n} Z_{i}$ and define $N(t)=\operatorname{Sup}\left\{n: S_{n} \leq t\right\}$. Then $N(t)$ counts the number of cycles completed in the interval $[0, t]$ and $M(t)=E[N(t)]$ is the renewal function associated with $\left\{Z_{n}\right\}$. By definition, $M(t)=\sum_{k=1}^{\infty} F_{Z}^{(k)}(t)$, where $F_{Z}^{(k)}(t)=P\left[S_{k} \leq t\right]$ is the $k$-fold convolution of $F_{Z}(t)$ and $F_{Z}(t)=F_{X} * F_{Y}(t)$, where * denotes the convolution operator. Now the expression for the interval reliability $R(x, t)$ can be written as

$$
R(x, t)=\bar{F}_{X}(t+x)+\int_{0}^{t} \bar{F}_{X}(t+x-u) d M(u), \text { where } \bar{F}_{X}(.)=1-F_{X}(.) .
$$

For example, when $F_{X}(t)=1-e^{-\lambda_{1} t}$ and $F_{Y}(t)=1-e^{-\lambda_{2} t}$, the interval reliability function is given by

$$
R(x, t)=\left[\frac{\lambda_{2}}{\lambda_{1}+\lambda_{2}}+\frac{\lambda_{1}}{\lambda_{1}+\lambda_{2}} e^{-\left(\lambda_{1}+\lambda_{2}\right) t}\right] e^{-\lambda_{1} x}=A(t) \bar{F}_{X}(x)
$$

Thus, when the sequences of failure and repair times are generated from two independent exponential distributions, the interval reliability function $R(x, t)$ is the product of the point availability function at time ' $t$ ', $A(t)$ and the reliability function at time ' $x$ ', $\bar{F}_{X}(x)$. In general, this may not be true. However, as a combined measure of availability and reliability, the interval reliability has a significant role in the study of repairable system performance.

We consider the nonparametric estimation of the interval reliability in this paper. The organization of the paper is as follows: Section 2 discusses the nonparametric estimation of the interval reliability based on complete observations. Section 3 discusses the estimation in the case of censored observations and in Section 4, we consider the estimation in the case of continuous observation over a fixed period. A simulation study is presented in Section 5 and an application of the proposed method is illustrated using a compressor failure data in Section 6.

\section{Estimation in the case of complete observations}

Suppose that observations on the failure times $X_{1}, X_{2}, \ldots, X_{n}$ and the repair times $Y_{1}, Y_{2}, \ldots, Y_{n}$ are available. Let $\hat{F}_{X}(t)$ and $\hat{F}_{Y}(t)$ denote the empirical distribution function of the random variables $X$ and $Y$ respectively. By definition

Published by Atlantis Press

Copyright: the authors 


$$
\hat{F}_{X}(t)=\frac{1}{n} \sum_{i=1}^{n} I_{\left(X_{i} \leq t\right)} \text { and } \hat{F}_{Y}(t)=\frac{1}{n} \sum_{i=1}^{n} I_{\left(Y_{i} \leq t\right)}
$$

where $I_{(B)}$ denotes the indicator function of an event $B$.

The estimation of the renewal function has been discussed extensively in the literature (cf., e.g., Frees 1986; Grubel and Pitts 1993; Harel et al. 1995). For fixed $t$, Baxter and Li (1994) proposed a method for constructing nonparametric confidence intervals for the renewal function which is easier to compute than that of Frees (1986). A natural estimator for the renewal function $M(t)$ is given by,

$$
\hat{M}_{n}(t)=\sum_{k=1}^{\infty} \hat{F}_{Z}^{(k)}(t)
$$

where $\hat{F}_{Z}(t)=\hat{F}_{X} * \hat{F}_{Y}(t)$.

However, in practical applications, we compute $\hat{M}_{n}(t)$ using the renewal equation, which is illustrated in Section 5.

We propose an estimator for the interval reliability as

$$
\hat{R}(x, t)=\hat{\bar{F}}_{X}(t+x)+\int_{0}^{t} \hat{\bar{F}}_{X}(t+x-u) d \hat{M}_{n}(u) .
$$

Baxter and $\mathrm{Li}$ (1994) studied asymptotic properties of the estimator $\hat{M}_{n}(t)$ defined by (1) and shown that $\hat{M}_{n}(t) \rightarrow M(t)$ almost surely as $n \rightarrow \infty$. By Glivenko-Cantelli Theorem, $\hat{F}_{X}(u) \rightarrow F_{X}(u)$ uniformly in $u$ as $n \rightarrow \infty$ with probability one. Hence, by Lemma 2.1 of Baxter and Li (1994), $\hat{R}(x, t) \rightarrow R(x, t)$ almost surely as $n \rightarrow \infty$ for any fixed $t$ and $x$.

Defining $J_{X}(t)=M * M * F_{X}(t), J_{Y}(t)=M * M * F_{Y}(t)$,

$$
\begin{aligned}
& V_{1}(x, t)=\int_{0}^{t} J_{Y}(t-u) d F_{X}(u+x), V_{2}(x, t)=\int_{0}^{t} J_{X}(t-u) d F_{Y}(u+x), \\
& K_{1}(x, u)=I_{(0<u<t)}\left[\bar{F}_{X}(x) J_{Y}(t-u)-V_{1}(x, t-u)\right]-I_{(0<u<x)}[M(t)+1]-I_{(x<u<t+x)}[M(t+x-u)+1], \text { and } \\
& K_{2}(x, u)=I_{(0<u<t)}\left[\bar{F}_{X}(x) J_{X}(t-u)-V_{2}(x, t-u)\right],
\end{aligned}
$$

we can write

$$
\sqrt{n}[\hat{R}(x, t)-R(x, t)]=\sqrt{n}\left(I_{1}+I_{2}+I_{3}\right),
$$

where $I_{1}=\int_{0}^{\infty} K_{1}(x, u) d \Delta F_{X}(u), \quad I_{2}=\int_{0}^{\infty} K_{2}(x, u) d \Delta F_{Y}(u)$, and $I_{3}$ contains terms of the form $\Delta A \Delta B, \Delta A * \Delta B$ and $\int_{0}^{t} \Delta A(u) d \Delta B(t+x-u)$

The derivation of the expression (2) is given in the Appendix.

By writing $\int_{0}^{t} \Delta A(u) d \Delta B(t+x-u)=\int_{0}^{t} \Delta A(u) d \hat{B}(t+x-u)-\int_{0}^{t} \Delta A(u) d B(t+x-u)$, it is easy to see that the two terms on the right-hand side converge almost surely to the same limit by using Lemma 2.1 of Baxter and Li (1994) and hence $\sqrt{n} I_{3} \rightarrow 0$ almost surely as $n \rightarrow \infty$.

Based on Donsker's Theorem and Helly-Bray Theorem (Billingsley, 1968) we have,

$$
\sqrt{n} I_{1} \stackrel{L}{\longrightarrow} \int_{0}^{\infty} K_{1}(x, u) d\left(W_{X}^{0} \circ F_{X}\right)(u)=\int_{0}^{1} K_{1}\left(x, F_{X}^{-1}(y)\right) d W_{X}^{0}(y),
$$

where $\left\{W^{0}(t), 0 \leq t \leq 1\right\}$ denotes a Brownian bridge and $\circ$ denotes functional composition.

Shorack and Wellner (1986, pp. 92-94) show that for Brownian bridge $\left\{W^{0}(t), 0 \leq t \leq 1\right\}$ and a square integrable function $h:[0,1] \rightarrow R, \int_{0}^{1} h(x) d W^{0}(x)$ is normally distributed with mean 0 and variance $\int_{0}^{1}[h(x)]^{2} d x-\left[\int_{0}^{1} h(x) d x\right]^{2}$. 
As an application of the above result, we get

$$
\sqrt{n} I_{1} \stackrel{L}{\longrightarrow} N\left(0, \sigma_{1}^{2}(x, t)\right) \text { as } n \rightarrow \infty,
$$

where

$$
\sigma_{1}^{2}(x, t)=\int_{0}^{\infty}\left[K_{1}(x, u)\right]^{2} d F_{X}(u)-\left[\int_{0}^{\infty} K_{1}(x, u) d F_{X}(u)\right]^{2} .
$$

Proceeding similarly as above, it can be shown that as $n \rightarrow \infty$,

$$
\sqrt{n} I_{2} \stackrel{L}{\longrightarrow} N\left(0, \sigma_{2}^{2}(x, t)\right),
$$

where

$$
\sigma_{2}^{2}(x, t)=\int_{0}^{\infty}\left[K_{2}(x, u)\right]^{2} d F_{Y}(u)-\left[\int_{0}^{\infty} K_{2}(x, u) d F_{Y}(u)\right]^{2} .
$$

Since $\Delta F_{X}$ and $\Delta F_{Y}$ are independent, $I_{1}$ and $I_{2}$ are also independent. This leads to the following theorem.

Theorem 1. For any fixed $\mathrm{t}$ and $\mathrm{x}$, as $n \rightarrow \infty$,

(i) $\hat{R}(x, t) \rightarrow R(x, t)$ almost surely and

(ii) $\sqrt{n}[\hat{R}(x, t)-R(x, t)] \stackrel{L}{\longrightarrow} N\left(0, \sigma^{2}(x, t)\right)$,

where $\stackrel{L}{\longrightarrow}$ denotes convergence in distribution and

$$
\sigma^{2}(x, t)=\sigma_{1}^{2}(x, t)+\sigma_{2}^{2}(x, t),
$$

with $\sigma_{1}^{2}(x, t)$ and $\sigma_{2}^{2}(x, t)$ are given by (3) and (4) respectively.

Remark. If we choose $x$ as 0 , then the estimator of the interval reliability, $\hat{R}(x, t)$, reduces to the estimator of the point availability, $\hat{A}(t)=\hat{R}(0, t)$ and the asymptotic properties of $\hat{A}(t)$ follow immediately from Theorem 1 by choosing $x=0$.

An estimator $\hat{\sigma}^{2}(x, t)$ of $\sigma^{2}(x, t)$ can be obtained on replacing $F_{X}(),. F_{Y}($.$) and M($.$) by \hat{F}_{X}(),. \hat{F}_{Y}($.$) and \hat{M}_{n}($.$) in$ (5) respectively. Using Lemma 2.1 of Baxter and $\mathrm{Li}(1994)$, it can be shown that $\hat{\sigma}^{2}(x, t) \rightarrow \sigma^{2}(x, t)$ almost surely as $n \rightarrow \infty$. Thus, given a significance level $\alpha \in(0,1)$, an approximate large sample $100(1-\alpha) \%$ confidence interval for $R(x, t)$ is

$$
\hat{R}(x, t)-z_{\alpha / 2} \frac{\hat{\sigma}(x, t)}{\sqrt{n}} \leq R(x, t) \leq \hat{R}(x, t)+z_{\alpha / 2} \frac{\hat{\sigma}(x, t)}{\sqrt{n}},
$$

where $z_{\alpha / 2}$ denotes the upper $\alpha / 2$ quantile of the standard normal distribution.

\section{Estimation in the case of censored observations}

Suppose that observations on the failure and repair time are subject to right censorship. In practice, a censored failure time occurs when the system is removed before failure for some preventive maintenance and a censored repair time occurs when the repair work is terminated before the repair is completed due to some technical reason. For example see Baxter and $\mathrm{Li}$ (1996) and $\mathrm{Li}$ (1999). Let $X_{1}, X_{2}, \ldots, X_{n}\left(Y_{1}, Y_{2}, \ldots, Y_{n}\right)$ denote the failure (repair) times and $C_{1}, C_{2}, \ldots, C_{n}\left(D_{1}, D_{2}, \ldots, D_{n}\right)$ denote the random censoring times associated with the failure (repair) times having distribution functions $F_{X}\left(F_{Y}\right)$ and $G_{C}\left(G_{D}\right)$ respectively. Suppose that the four random sequences $\left\{X_{i}\right\},\left\{Y_{i}\right\},\left\{C_{i}\right\}$ and $\left\{D_{i}\right\}$ are mutually independent and continuous. Under the censoring model, instead of observing $X_{i}$, we observe the pair $\left(T_{i}, \delta_{i}\right), i=1,2, \ldots, n$, where $T_{i}=\min \left(X_{i}, C_{i}\right)$ and $\delta_{i}=I_{\left(X_{i} \leq C_{i}\right)}$. Let $H_{X}()=.1-\left(1-F_{X}().\right)\left(1-G_{C}().\right)$ be the distribution function of $T_{i}$ and $\tau_{X}=\inf \left\{u: H_{X}(u)=1\right\} \leq \infty$ be the least upper bound for the support of $H_{X}$. With right-censored data, the most commonly used nonparametric estimator of $F_{X}(t)$ is the product limit estimator by Kaplan and Meier (1958):

$$
\hat{F}_{X, c}(t)=1-\prod_{i=1}^{n}\left[1-\frac{\delta_{(i)}}{n-i+1}\right]^{I_{\left(T_{(i)} \leq t\right)}} \text { for } t \leq T_{(n)} \text {, and } 1 \text { for } t>T_{(n)} \text {, }
$$


where $T_{(1)} \leq T_{(2)} \leq \ldots \leq T_{(n)}$ are the order statistics of $T_{1}, T_{2}, \ldots, T_{n}$ and $\delta_{(i)}$ denotes the concomitant associated with $T_{(i)}$. Similarly, we can construct the product limit estimator $\hat{F}_{Y, c}($.$) of F_{Y}($.$) . Let H_{Y}()=.1-\left(1-F_{Y}().\right)\left(1-G_{D}().\right)$ and $\tau_{Y}=\inf \left\{u: H_{Y}(u)=1\right\}$.

Let $\hat{M}_{c, n}(t)$ be an estimator of the renewal function $M(t)$ obtained by replacing $F_{X}$ and $F_{Y}$ with $\hat{F}_{X, c}$ and $\hat{F}_{Y, c}$ respectively. Then $\hat{M}_{c, n}(t)=\sum_{k=1}^{\infty} \hat{F}_{Z, c}^{(k)}(t)$, where $\hat{F}_{Z, c}(t)=\hat{F}_{X, c} * \hat{F}_{Y, c}(t)$.

In this case a nonparametric estimator of $R(x, t)$ is given by

$$
\hat{R}_{c}(x, t)=\hat{\bar{F}}_{X, c}(t+x)+\int_{0}^{t} \hat{\bar{F}}_{X, c}(t+x-u) d \hat{M}_{c, n}(u)
$$

$\mathrm{Li}$ (1999) discussed the nonparametric estimation of the renewal function with right-censored data and proved that $\hat{M}_{c, n}(t) \rightarrow M(t)$ almost surely as $n \rightarrow \infty$. Hence by the uniform convergence of $\hat{F}_{X, c}($.$) and Lemma 2.1$ of Baxter and $\mathrm{Li}$ (1994), it is easy to show that, for any fixed $t+x<\tau, \hat{R}_{c}(x, t) \rightarrow R(x, t)$ almost surely as $n \rightarrow \infty$.

In order to establish the weak convergence of $\hat{R}_{c}(x, t)$, let us define, $\Delta_{c} F_{X}(t)=\hat{F}_{X, c}(t)-F_{X}(t), \Delta_{c} F_{Y}(t)=\hat{F}_{Y, c}(t)-F_{Y}(t)$ and $\Delta_{c} M(t)=\hat{M}_{c}(t)-M(t)$.

By proceeding in the lines of the proof of Theorem 1, we can write

$$
\sqrt{n}\left[\hat{R}_{c}(x, t)-R(x, t)\right]=\sqrt{n} I_{1, c}+\sqrt{n} I_{2, c}+\sqrt{n} I_{3, c},
$$

where $I_{1, c}, I_{2, c}$, and $I_{3, c}$ are obtained by replacing $\Delta F_{X}(t)$ and $\Delta F_{Y}(t)$ with $\Delta_{c} F_{X}(t)$ and $\Delta_{c} F_{Y}(t)$ respectively in $I_{1}$, $I_{2}$ and $I_{3}$ defined in (2).

Following the same arguments used in Theorem 1, it can be shown that $\sqrt{n} I_{3, c} \rightarrow 0$ in probability as $n \rightarrow \infty$.

Fleming and Harrington (1990, p.235) proved that as $n \rightarrow \infty,\left\{\sqrt{n} \Delta_{c} F_{X}(t), t<\tau_{X}\right\}$ converges weakly to a Gaussian process $\left\{\bar{F}_{X}(t)\left(W_{X} \circ U_{X}\right)(t), t<\tau_{X}\right\}$, where $\left\{W_{X}(t), t \geq 0\right\}$ is a standard Brownian motion and $U_{X}(t)=\int_{0}^{t} \frac{d F_{X}(u)}{\bar{F}_{X}(u) \bar{H}_{X}(u)}$. As an application of this result with Helly-Bray Theorem,

Consider

$$
\begin{aligned}
\sqrt{n} I_{1, c}= & \sqrt{n} \int_{0}^{t+x} K_{1}(x, u) d \Delta_{c} F_{X}(u) \stackrel{L}{\longrightarrow} \int_{0}^{t+x} K_{1}(x, u) d\left\{\bar{F}_{X}(u)\left(W_{X} \circ U_{X}\right)(u)\right\} \\
& =\int_{0}^{t+x} K_{1}(x, u) \bar{F}_{X}(u) d\left(W_{X} \circ U_{X}\right)(u)+\int_{0}^{t+x} K_{1}(x, u)\left(W_{X} \circ U_{X}\right)(u) d \bar{F}_{X}(u) .
\end{aligned}
$$

$$
\begin{aligned}
& \int_{0}^{t+x} K_{1}(x, u)\left(W_{X} \circ U_{X}\right)(u) d \bar{F}_{X}(u)=\int_{0}^{t+x} K_{1}(x, u) \int_{0}^{u} d\left(W_{X} \circ U_{X}\right)(z) d \bar{F}_{X}(u) \\
& =\int_{0}^{t+x++x} \int_{z}^{t+x} K_{1}(x, u) d \bar{F}_{X}(u) d\left(W_{X} \circ U_{X}\right)(z)=\int_{0}^{t+x} P_{1}(x, z) d\left(W_{X} \circ U_{X}\right)(z),
\end{aligned}
$$

where $P_{1}(x, z)=\int_{z}^{t+x} K_{1}(x, u) d \bar{F}_{X}(u)$.

Thus, $\quad \sqrt{n} I_{1, c} \stackrel{L}{\longrightarrow} \int_{0}^{t+x}\left[K_{1}(x, u) \bar{F}_{X}(u)+P_{1}(x, u)\right] d\left(W_{X} \circ U_{X}\right)(u)$

$$
=\int_{0}^{U_{X}^{(t+x)}}\left[K_{1}\left(x, U_{X}^{-1}(y)\right) \bar{F}_{X}\left(U_{X}^{-1}(y)\right)+P_{1}\left(x, U_{X}^{-1}(y)\right)\right] d W_{X}(y) .
$$


Fleming and Harrington (1990, p.203) show that for Brownian motion $\{W(t), t \geq 0\}$ and a real, continuous function $h$, $\int_{0}^{t} h(x) d W(x)$ follows a normal distribution with mean 0 and variance $\int_{0}^{t}[h(x)]^{2} d x$.

Applying this result, we get, as $n \rightarrow \infty$

$$
\sqrt{n} I_{1, c} \stackrel{L}{\longrightarrow} N\left(0, \sigma_{1, c}^{2}(x, t)\right)
$$

where

$$
\sigma_{1, c}^{2}(x, t)=\int_{0}^{t+w}\left[K_{1}(x, u) \bar{F}_{X}(u)+P_{1}(x, u)\right]^{2} d U_{X}(u)
$$

Similarly we can show that as $n \rightarrow \infty$,

$$
\sqrt{n} I_{2, c} \stackrel{L}{\longrightarrow} N\left(0, \sigma_{2, c}^{2}(x, t)\right),
$$

where

$$
\sigma_{2, c}^{2}(x, t)=\int_{0}^{t}\left[K_{2}(x, u) \bar{F}_{Y}(u)+P_{2}(x, u)\right]^{2} d U_{Y}(u)
$$

with $U_{Y}(t)=\int_{0}^{t} \frac{d F_{Y}(u)}{\bar{F}_{Y}(u) \bar{H}_{Y}(u)}$, and $P_{2}(x, u)=\int_{u}^{t} K_{2}(x, y) d \bar{F}_{Y}(y)$.

By the independence of $I_{1, c}$ and $I_{2, c}$, we have the following theorem.

Theorem 2. For any fixed $t$ and $x$, as $n \rightarrow \infty$,

(i) $\hat{R}_{c}(x, t) \rightarrow R(x, t)$ almost surely for $t+x<\tau$, where $\tau=\min \left(\tau_{X}, \tau_{Y}\right)$

(ii) $\sqrt{n}\left[\hat{R}_{c}(x, t)-R(x, t)\right] \stackrel{L}{\longrightarrow} N\left(0, \sigma_{c}^{2}(x, t)\right)$ with

$$
\sigma_{c}^{2}(x, t)=\sigma_{1, c}^{2}(x, t)+\sigma_{2, c}^{2}(x, t),
$$

where $\sigma_{1, c}^{2}(x, t)$ and $\sigma_{2, c}^{2}(x, t)$ are given by (6) and (7) respectively.

In order to construct a consistent estimator of $\sigma_{c}^{2}(x, t)$, we use a consistent estimator $\hat{U}_{X}(t)$ of $U_{X}(t)$ proposed by Miller (1981) and it is given by,

$$
\hat{U}_{X}(t)=\sum_{i: T_{(i)} \leq t} \frac{\delta_{(i)}}{(n-i)(n-i+1)}, \text { where } \delta_{(i)} \text { is as defined before. }
$$

Similarly, an estimator $\hat{U}_{Y}(t)$ of $U_{Y}(t)$ can be constructed. On replacing $F_{X}(),. F_{Y}(),. U_{X}(),. U_{Y}($.$) by their$ corresponding consistent estimators in (8), a consistent estimator $\hat{\sigma}_{c}^{2}(x, t)$ of $\sigma_{c}^{2}(x, t)$ is obtained.

\section{Estimation in the case of continuous observation over a fixed period}

Suppose that the process is observed continuously over a fixed period $[0, T]$. Let $N_{X}(T)$ and $N_{Y}(T)$ denote the number of completed failures and repairs up to time $T$. Then the empirical estimators for the distribution functions $F_{X}(t)$ and $F_{Y}(t)$ can be defined as

$$
\hat{F}_{X, T}(t)=\frac{1}{N_{X}(T)} \sum_{i=1}^{N_{X}(T)} I_{\left(X_{i} \leq t\right)} \text { and } \hat{F}_{Y, T}(t)=\frac{1}{N_{Y}(T)} \sum_{i=1}^{N_{Y}(T)} I_{\left(Y_{i} \leq t\right)} .
$$

An estimator of the renewal function $M(t)$ in this case is given by,

$$
\hat{M}_{T}(t)=\sum_{k=1}^{\infty} \hat{F}_{Z, T}^{(k)}(t), \text { where } \hat{F}_{Z, T}(t)=\hat{F}_{X, T} * \hat{F}_{Y, T}(t) .
$$

As a nonparametric estimator of $R(x, t)$ we consider

$$
\hat{R}_{T}(x, t)=\hat{\bar{F}}_{X, T}(t+x)+\int_{0}^{t} \hat{\bar{F}}_{X, T}(t+x-u) d \hat{M}_{T}(u)
$$


The almost sure convergence of $\hat{F}_{X, T}(t)$ and $\hat{F}_{Y, T}(t)$ follows from the fact that both $N_{X}(T)$ and $N_{Y}(T)$ tend to infinity as $T \rightarrow \infty$. Thus it is straightforward to verify that $\hat{M}_{T}(t) \rightarrow M(t)$ almost surely and hence $\hat{R}_{T}(x, t) \rightarrow R(x, t)$ almost surely as $T \rightarrow \infty$.

Introducing the notation $\Delta_{T} A=\hat{A}_{T}-A$ and proceeding as in the case of complete observations, we can write,

$$
\sqrt{T}\left[\hat{R}_{T}(x, t)-R(x, t)\right]=\sqrt{T}\left(I_{1, T}+I_{2, T}+I_{3, T}\right),
$$

where $I_{1, T}, I_{2, T}$, and $I_{3, T}$ are obtained by replacing $\Delta$ by $\Delta_{T}$ in $I_{1}, I_{2}$, and $I_{3}$ respectively.

Following the arguments in Theorem 1 and using Lemma 3 stated in Ouhbi and Liminos (2003) it follows that $\sqrt{T} I_{3, T} \rightarrow 0$ in probability as $T \rightarrow \infty$.

Writing $\sqrt{T} I_{1, T}=\sqrt{\frac{T}{N_{X}(T)}} \sqrt{N_{X}(T)} I_{1, T}$ and using the fact that $N_{X}(T) / T \rightarrow 1 / \mu_{X}$ as $T \rightarrow \infty$, proceeding in the lines of the Theorem 1, we can show that $\sqrt{T} I_{1, T} \stackrel{L}{\longrightarrow} N\left(0, \mu_{X} \sigma_{1}^{2}(x, t)\right)$ and $\sqrt{T} I_{2, T} \stackrel{L}{\longrightarrow} N\left(0, \mu_{Y} \sigma_{2}^{2}(x, t)\right)$ as $T \rightarrow \infty$, where $\sigma_{1}^{2}(x, t)$ and $\sigma_{2}^{2}(x, t)$ are given in (3) and (4) respectively.

This leads to the following theorem.

Theorem 3. For any fixed $t$ and $x$, as $T \rightarrow \infty$,

(i) $\hat{R}_{T}(x, t) \rightarrow R(x, t)$ almost surely and

(ii) $\sqrt{T}\left[\hat{R}_{T}(x, t)-R(x, t)\right] \stackrel{L}{\longrightarrow} N\left(0, \sigma_{T}^{2}(x, t)\right)$,

where

$$
\sigma_{T}^{2}(x, t)=\mu_{X} \sigma_{1}^{2}(x, t)+\mu_{Y} \sigma_{2}^{2}(x, t) .
$$

On replacing $\mu_{X}, \mu_{Y}, F_{X}(),. F_{Y}($.$) and M($.$) by \bar{X}_{N_{X}(T)}, \bar{Y}_{N_{Y}(T)}, \hat{F}_{X, T}(),. \hat{F}_{Y, T}($.$) and \hat{M}_{T}($.$) respectively in (9) we get a$ consistent estimator $\hat{\sigma}_{T}^{2}(x, t)$ of $\sigma^{2}(x, t)$. Thus, given $\alpha \in(0,1)$, for large ' $T$ ', an approximate $100(1-\alpha) \%$ confidence interval for $R(x, t)$ is

$$
\hat{R}_{T}(x, t)-z_{\alpha / 2} \frac{\hat{\sigma}_{T}(x, t)}{\sqrt{T}} \leq R(x, t) \leq \hat{R}_{T}(x, t)+z_{\alpha / 2} \frac{\hat{\sigma}_{T}(x, t)}{\sqrt{T}} .
$$

\section{Simulation Study}

In this section we carry out an extensive simulation study to assess the finite sample performance of the proposed estimators in the case of i) complete observations, ii) censored observations and (iii) continuous observation over a fixed period. For computing the renewal function $\hat{M}(t)$, we consider an equally spaced partition of $[0, t]$, $0=t_{0}<t_{1}<\ldots<t_{m}=t$, where the choice of $m$ depends on $t$ and on the data. Now, $\hat{M}(t)$ is computed using the recursive relationship

$$
\hat{M}\left(t_{i}\right)=\hat{F}_{Z}\left(t_{i}\right)+\sum_{j=1}^{i} \hat{M}\left(t_{i}-t_{j}\right)\left[\hat{F}_{Z}\left(t_{j}\right)-\hat{F}_{Z}\left(t_{j-1}\right)\right], \text { for } i=1,2, \ldots, m .
$$

where $\hat{F}_{Z}\left(t_{i}\right)=\sum_{j=1}^{m} \hat{F}_{X}\left(t_{i}-t_{j}\right)\left[\hat{F}_{Y}\left(t_{j}\right)-\hat{F}_{Y}\left(t_{j-1}\right)\right]$.

For the case of complete observations, we assume that the distribution of the failure times is gamma with shape parameter 3 and scale parameter 2 and the repair times also follow a gamma distribution with shape parameter 1 and scale parameter 2. The time points $t=2.5,5$ and $x=0,0.5$ are considered for the simulation. The exact values of $R(x, t)$ at these points are obtained using Mathematica. The results of the simulation study are summarized in Table 1. Here ' $n$ ' denotes the number of completed cycles of the failure and repair times, $\hat{\bar{R}}(x, t)$ and $\hat{\bar{\sigma}}(x, t)$ denote the average of $\hat{R}(x, t)$ and $\hat{\sigma}(x, t)$ over 100 repetitions, and $R_{L}(x, t)$ and $R_{U}(x, t)$ denote the $95 \%$ lower and upper confidence limits for $R(x, t)$ respectively. The values given in the parenthesis represent the mean square error (MSE) of the corresponding estimator. 
Angel Mathew, N. Balakrishna

Table 1 Simulation results in the case of complete observations

\begin{tabular}{|c|c|c|c|c|c|c|c|}
\hline$t$ & $x$ & $n$ & $R(x, t)$ & $\hat{\bar{R}}(x, t)$ & $\hat{\bar{\sigma}}(x, t)$ & $R_{L}(x, t)$ & $R_{U}(x, t)$ \\
\hline \multirow[t]{4}{*}{2.5} & 0 & 25 & 0.84728 & $\begin{array}{l}0.83935 \\
(0.0645)\end{array}$ & $\begin{array}{l}0.39748 \\
(0.0635)\end{array}$ & 0.68354 & 0.99516 \\
\hline & & 100 & & $\begin{array}{l}0.84908 \\
(0.0341)\end{array}$ & $\begin{array}{l}0.40733 \\
(0.0294)\end{array}$ & 0.76924 & 0.92891 \\
\hline & 0.5 & 25 & 0.78454 & $\begin{array}{l}0.77501 \\
(0.0818)\end{array}$ & $\begin{array}{l}0.43977 \\
(0.0543)\end{array}$ & 0.60262 & 0.94739 \\
\hline & & 100 & & $\begin{array}{r}0.78022 \\
(0.0343) \\
\end{array}$ & $\begin{array}{r}0.44630 \\
(0.0255) \\
\end{array}$ & 0.69275 & 0.86770 \\
\hline \multirow[t]{4}{*}{5} & 0 & 25 & 0.75778 & $\begin{array}{l}0.74911 \\
(0.0720)\end{array}$ & $\begin{array}{l}0.51735 \\
(0.0219)\end{array}$ & 0.54631 & 0.95191 \\
\hline & & 100 & & $\begin{array}{c}0.75921 \\
(0.0478)\end{array}$ & $\begin{array}{l}0.52129 \\
(0.0100)\end{array}$ & 0.65704 & 0.86138 \\
\hline & 0.5 & 25 & 0.69385 & $\begin{array}{c}0.69972 \\
(0.0852)\end{array}$ & $\begin{array}{l}0.52903 \\
(0.0233)\end{array}$ & 0.49234 & 0.90710 \\
\hline & & 100 & & $\begin{array}{r}0.69323 \\
(0.0414) \\
\end{array}$ & $\begin{array}{l}0.53257 \\
(0.0109) \\
\end{array}$ & 0.58885 & 0.79762 \\
\hline
\end{tabular}

In order to check the performance of the estimator under censoring scheme we use the same distribution for the failure and repair times as in the case of complete observations. Further we assume that censoring distributions are exponential with cumulative distribution functions $G_{C}(t)=1-e^{-0.05 t}$ and $G_{D}(t)=1-e^{-0.1 t}$ respectively. The results of the simulation study are presented in Table 2 . Here $X \%$ and $Y \%$ denote the average percentage of censoring rate associated with the failure time and the repair time respectively.

Table 3 summarizes the result of the simulation study in the case of continuous observation over a fixed period $[0, T]$ using the same distributions for generating the failure and repair times as in the case of complete observations.

Here $\bar{N}(T)$ denotes the average number of cycles completed up to time ' $T$ '. From the simulation study, we see that even for moderate sample sizes, the proposed estimators perform well and the width of the confidence interval is reasonably narrow.

Table 2 Simulation results in the case of censored observations

\begin{tabular}{|c|c|c|c|c|c|c|c|c|c|}
\hline$t$ & $x$ & $n$ & $R(x, t)$ & $\hat{\bar{R}}_{c}(x, t)$ & $\hat{\bar{\sigma}}_{c}(x, t)$ & $X \%$ & $Y \%$ & $R_{L}(x, t)$ & $R_{U}(x, t)$ \\
\hline \multirow[t]{4}{*}{2.5} & 0 & 25 & 0.84728 & $\begin{array}{l}0.83986 \\
(0.0765)\end{array}$ & $\begin{array}{l}0.39047 \\
(0.0587)\end{array}$ & 22.88 & 16.16 & 0.68680 & 0.99292 \\
\hline & & 100 & & $\begin{array}{l}0.84564 \\
(0.0276)\end{array}$ & $\begin{array}{l}0.41556 \\
(0.0293)\end{array}$ & 24.72 & 17.52 & 0.76419 & 0.92709 \\
\hline & 0.5 & 25 & 0.78454 & $\begin{array}{l}0.79149 \\
(0.0631)\end{array}$ & $\begin{array}{l}0.42736 \\
(0.0561)\end{array}$ & 23.04 & 17.44 & 0.62396 & 0.95901 \\
\hline & & 100 & & $\begin{array}{l}0.78033 \\
(0.0315)\end{array}$ & $\begin{array}{l}0.45793 \\
(0.0220)\end{array}$ & 22.52 & 17.72 & 0.69058 & 0.87009 \\
\hline \multirow[t]{4}{*}{5} & 0 & 25 & 0.75778 & $\begin{array}{l}0.74990 \\
(0.0726)\end{array}$ & $\begin{array}{l}0.52929 \\
(0.0215)\end{array}$ & 22.56 & 19.68 & 0.54242 & 0.95738 \\
\hline & & 100 & & $\begin{array}{l}0.75363 \\
(0.0410)\end{array}$ & $\begin{array}{c}0.54908 \\
(0.0119)\end{array}$ & 23.40 & 17.60 & 0.64601 & 0.86125 \\
\hline & 0.5 & 25 & 0.69385 & $\begin{array}{l}0.69539 \\
(0.0858)\end{array}$ & $\begin{array}{l}0.54801 \\
(0.0494)\end{array}$ & 24.96 & 16.96 & 0.48057 & 0.91021 \\
\hline & & 100 & & $\begin{array}{l}0.69655 \\
(0.0379)\end{array}$ & $\begin{array}{l}0.55862 \\
(0.0160)\end{array}$ & 25.04 & 17.40 & 0.58706 & 0.80604 \\
\hline
\end{tabular}


Table 3 Simulation results in the case of continuous observation

\begin{tabular}{|c|c|c|c|c|c|c|c|c|}
\hline$t$ & $x$ & $T$ & $R(x, t)$ & $\hat{\bar{R}}_{T}(x, t)$ & $\hat{\bar{\sigma}}_{T}(x, t)$ & $\bar{N}(T)$ & $R_{L}(x, t)$ & $R_{U}(x, t)$ \\
\hline \multirow[t]{4}{*}{2.5} & 0 & 250 & 0.84728 & $\begin{array}{l}0.84345 \\
(0.0805)\end{array}$ & $\begin{array}{l}0.95755 \\
(0.1047)\end{array}$ & 31.44 & 0.72475 & 0.96215 \\
\hline & & 1000 & & $\begin{array}{l}0.84643 \\
(0.0288)\end{array}$ & $\begin{array}{l}0.98311 \\
(0.0584)\end{array}$ & 125.32 & 0.78550 & 0.90737 \\
\hline & 0.5 & 250 & 0.78454 & $\begin{array}{l}0.78330 \\
(0.0574)\end{array}$ & $\begin{array}{l}1.06905 \\
(0.0848)\end{array}$ & 30.40 & 0.65078 & 0.91583 \\
\hline & & 1000 & & $\begin{array}{r}0.78617 \\
(0.0394) \\
\end{array}$ & $\begin{array}{r}1.08486 \\
(0.0459) \\
\end{array}$ & 124.92 & 0.71893 & 0.85341 \\
\hline \multirow[t]{4}{*}{5} & 0 & 250 & 0.75778 & $\begin{array}{l}0.76089 \\
(0.0711)\end{array}$ & $\begin{array}{l}1.25714 \\
(0.0732)\end{array}$ & 31.84 & 0.60505 & 0.91673 \\
\hline & & 1000 & & $\begin{array}{l}0.75889 \\
(0.0338)\end{array}$ & $\begin{array}{c}1.28293 \\
(0.0304)\end{array}$ & 123.28 & 0.67937 & 0.83841 \\
\hline & 0.5 & 250 & 0.69385 & $\begin{array}{l}0.68660 \\
(0.0865)\end{array}$ & $\begin{array}{l}1.28439 \\
(0.1125)\end{array}$ & 31.56 & 0.52739 & 0.84582 \\
\hline & & 1000 & & $\begin{array}{l}0.69146 \\
(0.0336)\end{array}$ & $\begin{array}{c}1.30958 \\
(0.0294)\end{array}$ & 124.00 & 0.61029 & 0.77263 \\
\hline
\end{tabular}

\section{An example of application}

We carry out a data analysis to illustrate an application of the proposed estimation procedure using compressor failure data given in Table 7.1 and Table 11.7 of Rausand and Høyland (2004). The data consists of the operating and repair times of 90 critical failures of a specific compressor at a Norwegian process plant in the time period from 1968 until 1989. In the given data set the failure times are measured in days and the repair times are measured in hours. For the meaningful computation purpose we convert the failure time data to hours and the interval reliability $R(x, t)$ is computed with $t=15,20,25$ and $x=0,2.5,5,7.5$ hours. The $95 \%$ confidence intervals are also computed for the interval reliability at these time points and are summarized in Table 4. Here, the upper limit of the confidence interval for the interval reliability, $R_{U}(x, t)$, is greater than 1 at several time points because the estimated value of the interval reliability at these time points is near to its maximum value 1 . So, if $R_{U}(x, t)$ is greater than 1 , it is replaced by 1 in the Table to make meaningful interpretation.

Table 4 Interval reliability computation of compressor failure data

\begin{tabular}{cccccc}
\hline$t$ & $x$ & $\hat{\bar{R}}(x, t)$ & $\hat{\sigma}(x, t)$ & $R_{L}(x, t)$ & $R_{U}(x, t)$ \\
\hline 15 & 0 & 0.97716 & 0.36676 & 0.90139 & 1.00000 \\
& 2.5 & 0.97138 & 0.38039 & 0.89279 & 1.00000 \\
& 5 & 0.96710 & 0.39054 & 0.88641 & 1.00000 \\
& 7.5 & 0.96564 & 0.39399 & 0.88424 & 1.00000 \\
\hline 20 & 0 & 0.97330 & 0.38119 & 0.89454 & 1.00000 \\
& 2.5 & 0.97166 & 0.38531 & 0.89206 & 1.00000 \\
& 5 & 0.89060 & 0.44750 & 0.79814 & 0.98305 \\
& 7.5 & 0.88750 & 0.45354 & 0.79380 & 0.98120 \\
\hline 25 & 0 & 0.92945 & 0.43896 & 0.83876 & 1.00000 \\
& 2.5 & 0.92560 & 0.44657 & 0.83334 & 1.00000 \\
& 5 & 0.92123 & 0.45485 & 0.82726 & 1.00000 \\
& 7.5 & 0.91884 & 0.45956 & 0.82390 & 1.00000 \\
\hline
\end{tabular}




\section{Concluding remarks}

We have discussed the nonparametric estimation of the interval reliability when the operating and repair times of a system are mutually independent sequences of i.i.d. random variables. The proposed estimators of the interval reliability are proved to be consistent and asymptotically normal when (i) the data are complete, (ii) the data are subject to right censorship and (iii) the data are observed over a fixed period. The simulation study shows that the proposed estimators perform well even for reasonable sample sizes. Finally the estimation procedure corresponding to the complete sample is illustrated using a real life data.

\section{References}

1. Abraham, B., and Balakrishna, N. (2000). Estimation of limiting availability for a stationary bivariate process. Journal of Applied Probability, 37, 696-704.

2. Balakrishna, N., and Mathew, A. (2009). Nonparametric estimation of the average availability. Communications in Statistics Theory and Methods, 38, 1207-1218.

3. Barlow, R.E., and Hunter, L.C. (1961). Reliability analysis of a one unit system, Operations Research, 9, 200-208.

4. Barlow, R.E., and Proschan, F. (1975). Statistical Theory of Reliability and Life Testing. Rinehart and Winston, New York.

5. Baxter, L.A.,, and Li, L. (1994). Nonparametric confidence intervals for the renewal function and the point availability. Scandinavian Journal of Statistics, 21, 277-287.

6. Baxter, L.A., and Li, L. (1996). Nonparametric estimation of the limiting availability. Lifetime Data Analysis, 2, 391-402.

7. Billingsley, P. (1968). Convergence of Probability Measures. Wiley, New York.

8. Fleming, T. R., and Harrington, D. P. (1991). Counting Processes and Survival Analysis, Wiley, New York.

9. Frees, E.W. (1986). Warranty analysis and renewal function estimation. Naval Research Logistics Quarterly, 33, 361-372.

10. Grubel, R., and Pitts, S.M. (1993). Nonparametric estimation in renewal theory I: The empirical renewal function. The Annals of Statistics, 21, 1431-1451.

11. Harel, M., O'Cinneide, C.A., and Schneider, H. (1995). Asymptotics of the sample renewal function. Journal of Mathematical Analysis and Applications, 189, 240-255.

12. Kaplan, E.L., and Meier, P. (1958). Nonparametric estimation from incomplete observations. Journal of the American Statistical Association, 53, 457-481.

13. Li, L. (1999). Estimating the point availability with right censored data. Naval Research Logistics, 46, 119-127.

14. Mi, J. (1995). Limiting behaviour of some measures of system availability. Journal of Applied Probability, 32, $482-493$.

15. Mi, J. (1999). On measure of system interval availability, Probability in the Engineering and Informational Sciences, 13, 359-375.

16. Miller, R.G. (1981). Survival Analysis. John Wiley \& Sons, New York.

17. Nakagawa, T. (2005). Maintenance Theory of Reliability. Springer-Verlag, London.

18. Ouhbi, B., and Liminios, N. (2003). Nonparametric Reliability Estimation of Semi-Markov Processes. Journal of Statistical Planning and Inference, 109, 155-165.

19. Rausand, M., and Høyland, A. (2004). System Reliability Theory: Models, Statistical Methods, and Applications. John Wiley \& Sons, New Jersey.

20. Shorack, G.R., and Wellner, J.A. (1986). Empirical Process with Applications to Statistics. John Wiley and Sons, New York.

\section{Appendix A. Derivation of expression (2)}

We can write

$$
\begin{aligned}
\Delta R(x, t) & =\hat{R}(x, t)-R(x, t) \\
& =\hat{\bar{F}}_{X}(t+x)-\bar{F}_{X}(t+x)+\int_{0}^{t} \hat{\bar{F}}_{X}(t+x-u) d \hat{M}(u)-\int_{0}^{t} \bar{F}_{X}(t+x-u) d M(u) \\
& =\Delta \bar{F}_{X}(t+x)+\Delta\left[\int_{0}^{t} \bar{F}_{X}(t+x-u) d M(u)\right] \\
& =\Delta \bar{F}_{X}(t+x)+\Delta\left[\bar{F}_{X}(x) M(t)+\int_{0}^{t} M(u) d F_{X}(t+x-u)\right] .
\end{aligned}
$$

Now using the fact that $\Delta[A B]=\hat{A} \hat{B}-A B=\Delta A \Delta B+A \Delta B+B \Delta A$, we can write 
$\Delta R(x, t)=\Delta \bar{F}_{X}(t+x)+M(t) \Delta \bar{F}_{X}(x)+\bar{F}_{X}(x) \Delta M(t)+\int_{0}^{t} \Delta M(u) d F_{X}(t+x-u)+\int_{0}^{t} M(u) d \Delta F_{X}(t+x-u)+Q_{1}$,

where $Q_{1}=\Delta \bar{F}_{X}(x) \Delta M(t)+\int_{0}^{t} \Delta M(u) d \Delta F_{X}(t+x-u)$.

Following the equation (2.2) of Harel et al. (1995), we can write

$$
\Delta M(t)=M * M * \Delta F_{Z}(t)+\Delta F_{Z} * \Delta F_{Z} * \hat{M} * M(t),
$$

where $\Delta F_{Z}(t)=\Delta F_{X} * \Delta F_{Y}+F_{X} * \Delta F_{Y}+F_{Y} * \Delta F_{X}$

Thus

$$
\Delta M(t)=M * M * F_{Y} * \Delta F_{X}(t)+M * M * F_{X} * \Delta F_{Y}(t)+\Delta Q(t)=J_{Y} * \Delta F_{X}(t)+J_{X} * \Delta F_{Y}(t)+\Delta Q(t),
$$

where $\Delta Q(t)=M * M * \Delta F_{X} * \Delta F_{Y}(t)+\Delta F_{Z} * \Delta F_{Z} * \hat{M} * M(t)$.

Substituting for $\Delta M(t)$ in $\Delta R(x, t)$, we get

$$
\begin{aligned}
\Delta R(x, t)=\Delta \bar{F}_{X}(t+x)+M(t) \Delta \bar{F}_{X}(x)+\bar{F}_{X}(x) J_{Y} * \Delta F_{X}(t)+\bar{F}_{X}(x) J_{X} * \Delta F_{Y}(t) & \\
& +\int_{0}^{t} J_{Y} * \Delta F_{X}(u) d F_{X}(t+x-u)+\int_{0}^{t} J_{X} * \Delta F_{Y}(u) d F_{X}(t+x-u)+\int_{0}^{t} M(u) d \Delta F_{X}(t+x-u)+Q_{1}+Q_{2},
\end{aligned}
$$

where $Q_{2}=\Delta \bar{F}_{X}(x) \Delta Q(t)+\int_{0}^{t} \Delta Q(u) d \Delta F_{X}(t+x-u)$.

Now,

$$
\Delta R(x, t)=I_{1}+I_{2}+I_{3},
$$

where

$$
\begin{gathered}
I_{1}=\Delta \bar{F}_{X}(t+x)+M(t) \Delta \bar{F}_{X}(x)+\bar{F}_{X}(x) J_{Y} * \Delta F_{X}(t) \\
+\int_{0}^{t} J_{Y} * \Delta F_{X}(u) d F_{X}(t+x-u)+\int_{0}^{t} M(u) d \Delta F_{X}(t+x-u), \\
I_{2}=\bar{F}_{X}(x) J_{X} * \Delta F_{Y}(t)++\int_{0}^{t} J_{X} * \Delta F_{Y}(u) d F_{X}(t+x-u) \text { and } I_{3}=Q_{1}+Q_{2} .
\end{gathered}
$$

Since $\int_{0}^{t} J_{Y} * \Delta F_{X}(u) d F_{X}(t+x-u)=\int_{0}^{t} \int_{0}^{u} J_{Y}(u-y) d \Delta F_{X}(y) d F_{X}(t+x-u)$

$$
=\int_{0}^{t} \int_{y}^{t} J_{Y}(u-y) d F_{X}(t+x-u) d \Delta F_{X}(y)=-\int_{0}^{t} V_{1}(x, t-y) d \Delta F_{X}(y),
$$

$I_{1}$ can be written as

$$
\begin{aligned}
I_{1} & =-\int_{0}^{t+x} d \Delta F_{X}(u)-M(t) \int_{0}^{x} d \Delta F_{X}(u)+\bar{F}_{X}(x) \int_{0}^{t} J_{Y}(t-u) d \Delta F_{X}(u) \\
& \quad-\int_{0}^{t} V_{1}(x, t-u) d \Delta F_{X}(u)-\int_{x}^{t+x} M(t+x-u) d \Delta F_{X}(u) \\
= & \int_{0}^{\infty} K_{1}(x, u) d \Delta F_{X}(u) .
\end{aligned}
$$

Similarly, we can write $I_{2}=\int_{0}^{\infty} K_{2}(x, u) d \Delta F_{Y}(u)$. 